\title{
Spatially resolved measurements of electrochemically induced spillover on porous and microstructured Pt/YSZ catalysts
}

\author{
R. Imbihl*, J. Janek \\ Institut für Physikalische Chemie und Elektrochemie, Universität Hannover, Callinstr. 3-3a, D-30167 Hannover, Germany
}

\begin{abstract}
$\mathrm{Pt} / \mathrm{YSZ}$ electrodes prepared from platinum (Pt) and yttria-stabilized zirconia (YSZ) can be applied in electrocatalytic experiments for the promotion of different heterogeneously catalyzed reactions. The strong increase in catalytic activity of Pt/YSZ assemblies on electrochemical polarisation has recently been attributed to the creation of catalytically active spill-over species under non-equilibrium conditions. Our experiments combine locally resolved surface sensitive techniques (SPEM, PEEM) with electrochemical methods in order to resolve the processes at the Pt/YSZ interface and to clarify the nature of the spillover species.
\end{abstract}

Keywords: Solid electrolyte; Electrocatalysis; YSZ; Platinum; Photoelectron emission microscopy

\section{Introduction}

The idea of an electrochemical promotion of an heterogeneously catalyzed reaction has been proposed nearly three decades ago by Wagner [1]. A very efficient electrochemical promotion of heterogeneously catalyzed reactions has been achieved by the electrochemical polarisation of porous metal films deposited on solid electrolytes [2-6]. Although this so-called NEMCA effect (non-Faradaic electrochemical modification of catalytic activity) pioneered by Vayenas et al. [2-6] has up to now been demonstrated for over 40 reactions employing different solid electrolytes and a variety of different metal electrodes a detailed mechanistic picture is still

*Corresponding author. Tel.: +49-511-762-2349; fax: +49511-762-4009.

E-mail addresses: imbihl@mbox.pci.uni-hannover.de (R. Imbihl),janek@mbox.pci.uni-hannover.de (J. Janek). lacking. In particular, the nature of the spillover species which has been postulated to explain the observed changes in work function and catalytic activity has not been clarified unambiguously.

Our aim is to employ the concept of surface science and to combine surface sensitive methods with electrochemical measurements in order to obtain a clear picture of the mechanism of electrocatalysis. We therefore use surface sensitive techniques to characterize catalyst samples in an ultrahigh vacuum (UHV) environment and to follow their catalytic behavior in measurements under low pressure reaction conditions in situ. Ideally, one should be able to correlate characteristics in the electrochemical measurements with changes in the state of the surface and in the catalytic behavior.

In order to reduce the complexity of the system we construct model catalysts using lithographic techniques to prepare an yttrium stabilized zirconia (YSZ) sample with a thin microstructured Pt film on 
top (see Fig. 1). In this way we obtain a well defined interface Pt/YSZ accessible to experimental observation. We employ PEEM (photoelectron emission microscopy) and SPEM (scanning photoelectron microscopy) as spatially resolving techniques to follow the spreading and distribution of an electrochemically induced spillover species and we will correlate spatially resolving in situ measurements with integral reaction rates [7-10].

With this type of concept a pressure gap exists between the NEMCA experiments conducted typically under atmospheric pressure and our surface science experiments carried out in a pressure range from $\approx 10^{-10}$ to $10^{-3}$ mbar. In order to be able to relate our findings to the NEMCA effect it is necessary to bridge this pressure gap and to complement the surface science experiments by experiments

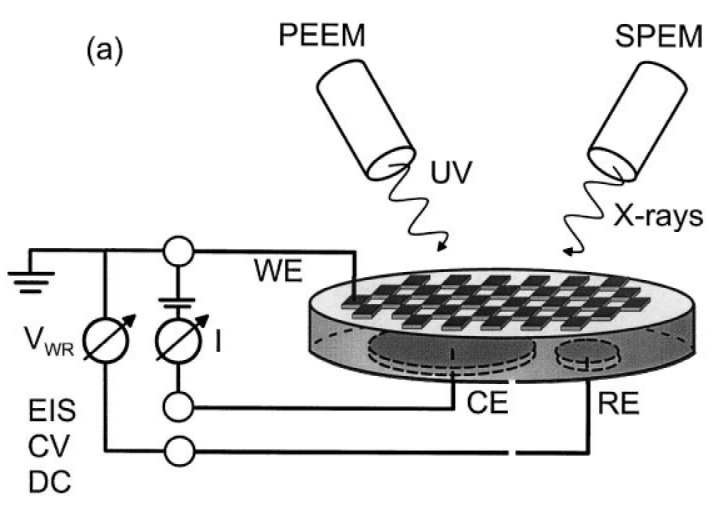

(b)

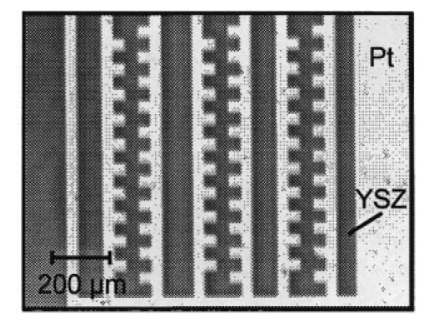

Fig. 1. (a) Schematic drawing of the experimental set-up showing the single crystalline YSZ sample $(d=1 \mathrm{~mm}, 20 \mathrm{~mm}$ diameter) with the microstructured $500 \AA \mathrm{Pt}$ film as working electrode on one side and the reference and the counter electrode both prepared with Ag paste on the other side. (b) Optical micrograph of the characteristic structure of the microstructured $\mathrm{Pt}$ film on single crystalline YSZ used for the SPEM experiments. in the intermediate pressure range. Such experiments are underway.

In surface science studies of heterogeneously catalyzed reactions one faces typically a second kind of fundamental problem besides the above mentioned pressure gap which is the 'materials gap' between single crystal studies and real catalysts. A similar problem exists also in electrocatalysis because the structural and chemical properties of a microstructured catalyst will of course differ very strongly from the properties of typical polycrystalline YSZ samples with porous sintered Pt layers prepared by calcination of a Pt paste. However, here one faces a more complicated materials problem: Even when one compares samples with porous sintered Pt films on polycrystalline YSZ but prepared by different groups their properties differ drastically. This demonstrates that the materials problem is in fact significant and that systematic studies have to be undertaken in order to clarify the influence of the preparation on the electrocatalytic properties of the samples.

In the following we present a number of experiments showing how spatially resolving techniques in combination with electrochemical methods can be used to gain detailed information about processes that play an essential role in the electrochemical promotion of reactions. We look at the $\mathrm{Pt} / \mathrm{YSZ}$ interface and we find that upon electrochemical pumping, chemical changes are induced on the $\mathrm{Pt}$ surface as well as on the zirconia surface.

\section{Experimental methods}

In Fig. 1a the typical experimental setup is depicted schematically. The catalyst film on top of the electrolyte is used as the working electrode (WE) in a conventional three electrode arrangement. Two other metal films on the opposite side are used as counter electrode (CE, silver) and reference electrode (RE, silver). A controlled potential $V_{\mathrm{WR}}$ is applied to the catalyst film, and the corresponding electric current $I$ is measured between WE and CE. With an EG\&G potentiostat and an impedance analyser all standard electrochemical methods can be applied. Thus, catalyst film/YSZ electrodes can be characterized by their DC characteristics, their impedance spectra (EIS) in the range from $1 \mathrm{mHz}$ to $1 \mathrm{MHz}$ and 
by cyclic voltammetry $(\mathrm{CV}$, linear sweep voltammetry in continuous cycles) both under UHV and under atmospheric conditions. Additionally, a Kelvin probe is available in the UHV chamber. Under open cell conditions $(I=0), V_{\mathrm{WR}}$ is a measure of the chemical potential difference of oxygen between catalyst film and reference electrode. Under UHV conditions, both sides of the samples are exposed to the gas atmosphere of the UHV chamber. Thus, $V_{\mathrm{wR}}$ should equal zero under opencell conditions. Any non-zero value of $V_{\mathrm{WR}}$ under open cell conditions corresponds to chemical polarisation of the catalyst film (ignoring small thermovoltages due to a temperature gradient across the sample).

In PEEM experiments one illuminates the sample with photons from a $\mathrm{D}_{2}$ discharge lamp (6 eV emission maximum) [7]. Collecting the emitted photoelectrons one essentially images the local work function with a spatial resolution of $0.1-1 \mu \mathrm{m}$ and the temporal resolution of video frames $(40 \mathrm{~ms})$. The WE is connected to ground so that no charging up should occur during such measurements. SAES (scanning auger electron microscopy) was used for controlling the chemical composition of the surface. Monitoring a varying oxygen coverage with SAES turned out to be difficult due to beam damaging effects. The samples were radiatively heated from the backside by a $240-\mathrm{W}$ lamp. The oxygen partial pressure can be varied between the background pressure of $10^{-8}$ and $10^{-3}$ mbar.

We investigated three types of Pt/YSZ samples: (i) samples with a porous Pt layer on polycrystalline YSZ supplied by the Patras group, (ii) samples with a porous $\mathrm{Pt}$ layer on single crystalline or polycrystalline YSZ prepared in our laboratory, and (iii) microstructured Pt/YSZ samples shown in Fig. 1b fabricated with photolithographic techniques by evaporation of a 500- $\AA$ thick Pt film onto a single crystalline YSZ sample.

\section{Pt/YSZ samples with a porous Pt film}

We studied polycrystalline samples with porous sintered Pt films supplied by the Patras group and compared their behavior with that of samples prepared in our laboratory. Surprisingly, we found that despite nearly identical electrochemical characteris- tics the two types of samples differed drastically in their electrocatalytic properties. An essential finding of all the previous NEMCA experiments has been that the application of an electric potential to the working electrode (WE) leads to a corresponding work function change on the WE as expressed by the equality, $\Delta \phi=V_{\mathrm{WR}}$ [11]. Whereas the Pt electrodes of the samples prepared in our laboratory exhibited no detectable WF changes at all upon electrochemical pumping (detection limit of the Kelvin probe $\approx 10 \mathrm{mV}$ ), the WF of the Pt electrodes on the Patras samples followed the variation of the potential $V_{\mathrm{WR}}$ in a range from -2 to $+2 \mathrm{~V}$ as predicted by the above mentioned equality. Both types of samples showed an electrocatalytic effect in catalytic $\mathrm{CO}$ oxidation but again the quantitative and even qualitative behavior was different [7]. However, for both types of samples the electrocatalytic rate increase was always faradaic within the accuracy of our measurements (estimated to $50 \%$ of the reaction rate). This means that in the pressure range from $10^{-8}$ to $10^{-4}$ mbar range covered by our measurements we could not find a NEMCA effect.

$\mathrm{CV}$ measurements under low pressure conditions with the same sample give results which are apparently similar to those of Vayenas' group [11,12] (Fig. 2. Sweeping $V_{\mathrm{WR}}$ from a positive potential $(+0.5 \mathrm{~V})$ to a negative potential $(-0.5 \mathrm{~V})$, a pronounced peak structure with at least two minima can be observed (at different oxygen pressures between $10^{-8}$ and $10^{-4}$ mbar and at different sweep rates between 10 and $100 \mathrm{mV} \mathrm{s}^{-1}$ ). The appearance of these minima changes drastically with prolonged oxygen pumping (pump time $t_{\mathrm{p}}$ ) at $V_{\mathrm{WR}}=+0.5 \mathrm{~V}$, i.e. the dominating minimum is shifted to more positive potentials and becomes more narrow with a higher peak current. Thus, upon oxygen pumping, oxygen species are created anodically at the electrode interface and are reduced during the cathodic sweep. If a second or further sweep (without pumping at $0.5 \mathrm{~V}$ again) is performed after the first sweep, the $\mathrm{CV}$ become identical, only showing a single minimum. The occurrence of at least two minima is interpreted by Vayenas by the existence of chemisorbed oxygen and an oxygen spillover species. During the anodic sweep from negative to positive potentials no peak at all is observed, thus indicating the strongly irreversible character of the electrode processes. Despite the 


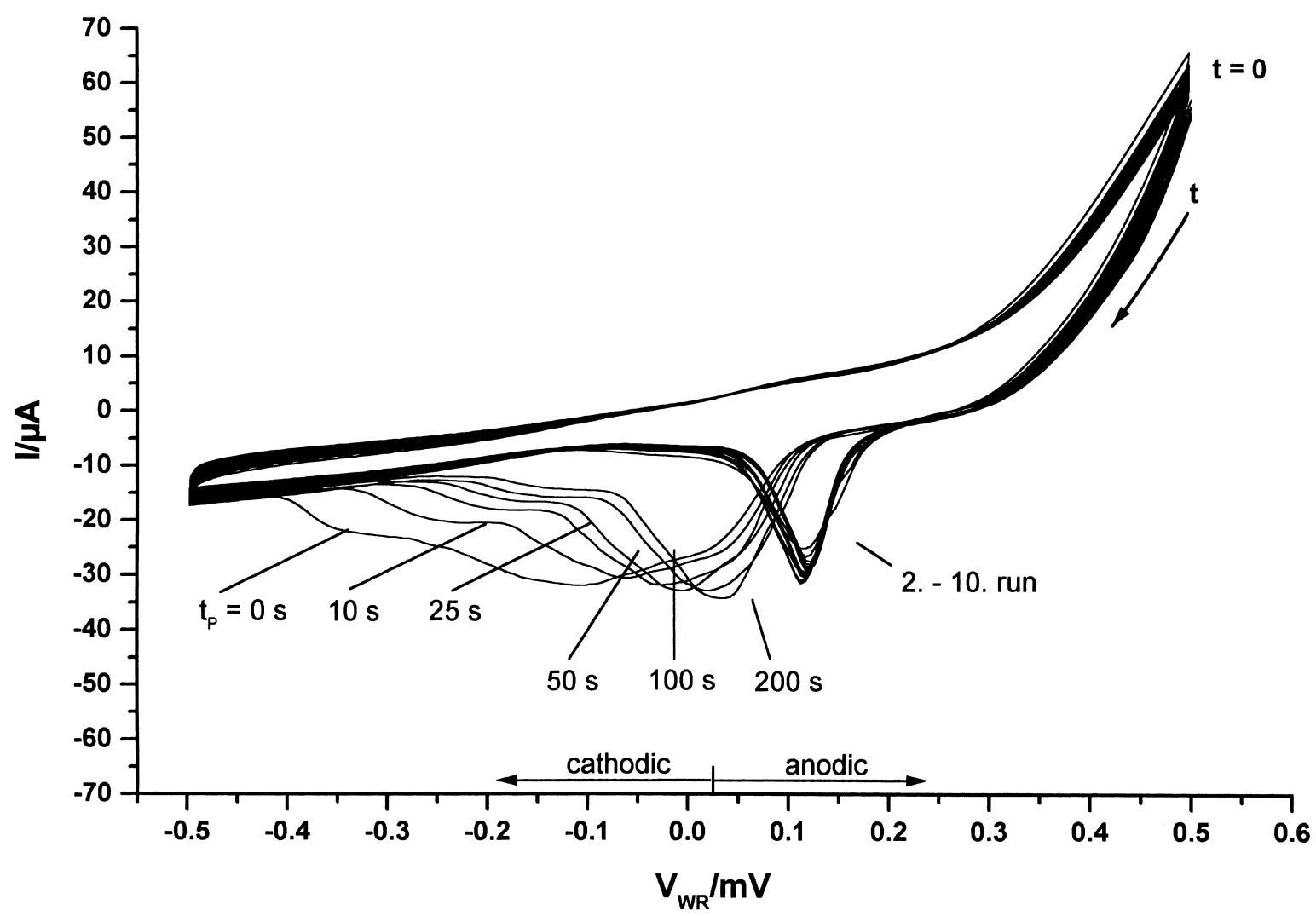

Fig. 2. Cyclovoltammograms (CV) of the Pt/YSZ electrode (Patras sample) which was used for the PEEM measurements in Fig. 3 under UHV conditions. Before each CV run, oxygen was pumped anodically for the specified time interval $\left(t_{\mathrm{p}}=0-200 \mathrm{~s}\right)$. Experimental conditions: $p=10^{-5}$ mbar, $T=773 \mathrm{~K}$, sweep rate $=10 \mathrm{mV} \mathrm{s}^{-1}$.

close similarity between the CV results of Vayenas and us, a surprising difference has to be noted. Whereas we observe a shift of the minima to more anodic potentials on pumping, Vayenas observes a shift to more cathodic potentials. Further experiments have to clarify this difference.

In spatially resolved measurements with PEEM we found that the WF changes which can be observed on the Patras samples upon electrochemical pumping always occurred spatially homogeneous on the $\mathrm{Pt}$ electrodes [13]. In particular, we found no sources from where the spreading of a spillover species took place via fronts. At the boundary Pt/YSZ we observed, however, that upon cathodic pumping with negative potential $V_{\mathrm{WR}}$ a reduction front spreads out onto the YSZ surface starting at the Pt/YSZ interface. Thus, the YSZ electrolyte itself is reduced which leads to an decrease of the work function.
This process is demonstrated in the PEEM images displayed in Fig. 3a-c. The reverse process, the increase of the work function, i.e. the darkening of the YSZ surface in PEEM upon application of a positive (anodic) potential $V_{\mathrm{WR}}$ takes place spatially uniformly. We can assume that such changes will also occur at the three phase boundary hidden beneath the porous Pt layer which plays an essential role in the formation of spillover species and in the NEMCA effect. A correlation between work function changes and the current minima in CV could so far not be substantiated.

\section{Microstructured Pt/YSZ samples}

In order to have the three-phase-boundary (tpb) of the Pt/YSZ samples accessible to direct observation 


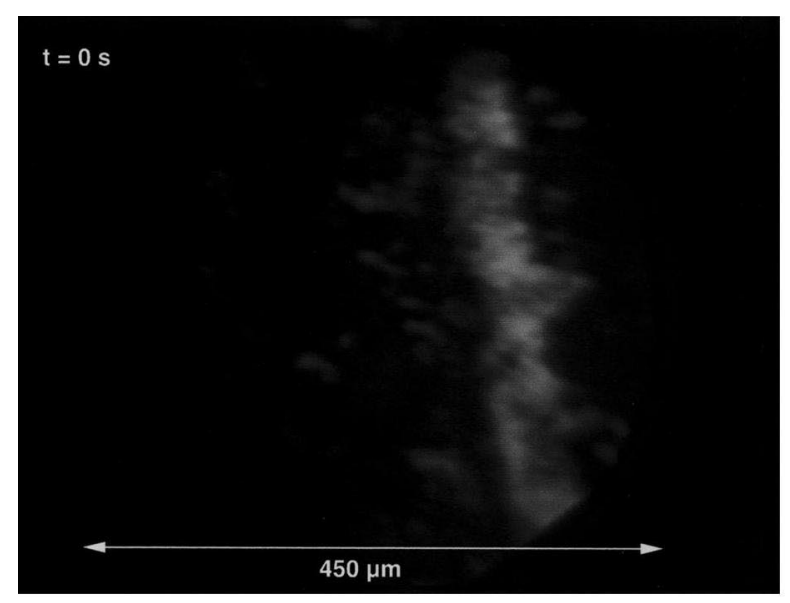

(a)

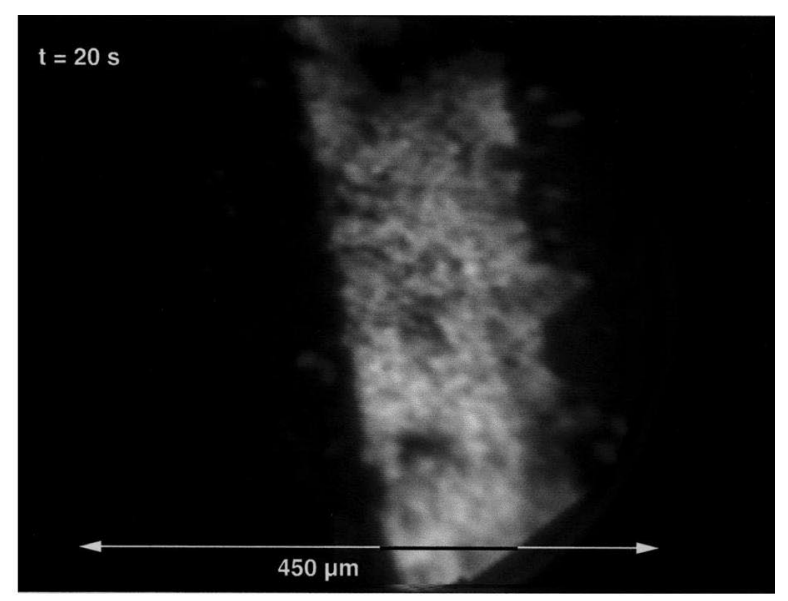

(b)

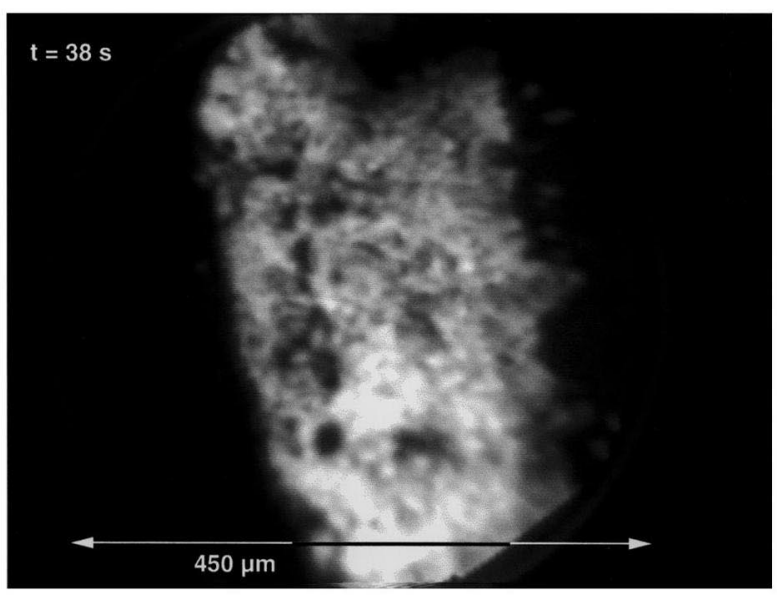

(c)

Fig. 3. PEEM images at three different times showing the spreading (from right to left) of a reduction front on the polycrystalline YSZ surface from the boundary of a porous Pt electrode (dark area on the right side) upon electrochemical pumping with negative potential $V_{\mathrm{WR}}$ (reduction). Bright areas correspond to low work function, dark areas to high work function. A Pt/YSZ sample prepared by the Patras group was used in the experiment. Experimental conditions: background pressure $\approx 10^{-8} \mathrm{mbar}, T=673 \mathrm{~K}, V_{\mathrm{WR}}=-0.5 \mathrm{~V}$ (from Ref. [13]).

we prepared microstructured Pt/YSZ catalysts with $500-\AA$ thick Pt films employing optical lithography (see Fig. 1). In PEEM measurements of the microstructured Pt/YSZ samples we observed work function changes of the Pt surface upon electrochemical pumping which we can attribute to the formation of a spillover species [7]. As shown by the PEEM images in Fig. 4 a darkening of the Pt surface upon applying a positive voltage $V_{\mathrm{WR}}$ and a brightening with a negative voltage $V_{\mathrm{WR}}$ was seen. These surface changes correspond to a work function increase and a work function decrease, respectively. No time dependent spatial concentration profiles were registered which would have been indicative of the slow diffusional spreading of a spillover species. Such a spreading should start from the tpb, i.e. from the edges of the Pt film. This, however, was not seen and possible reasons can either be a diffusion too fast for our time resolution $(40 \mathrm{~ms})$ or diffusion via cracks and grain boundaries in the Pt film giving within the 

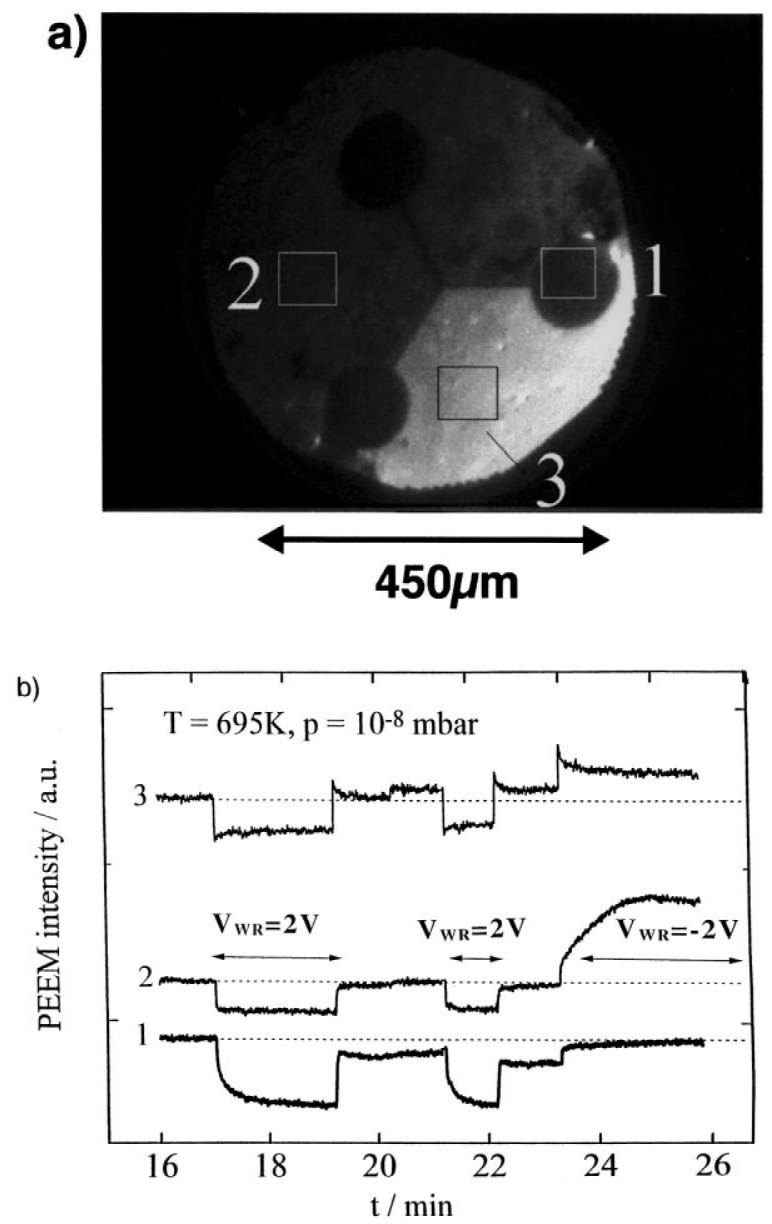

Fig. 4. Electrochemically induced surface changes on a Pt/YSZ micro-structure (from Ref. [7]). (a) PEEM image of the Pt/YSZ microstructure showing three circular YSZ domains connected via channels which are surrounded by a Pt film. Inside the windows marked from 1 to 3 , the digitized PEEM intensity has been integrated for the measurements displayed in (b). The lower one of the three Pt segments is obviously not in electric contact with the other two segments and therefore exhibits a different electrochemical behavior upon electrochemical pumping. (b) Local brightness variations in the windows marked in (a) during electrochemical pumping at $T=695 \mathrm{~K}$. The background pressure was about $10^{-8} \mathrm{mbar}$ (at $V_{\mathrm{WR}}=0 \mathrm{~V}$ ).

resolution of PEEM $(\approx 1 \mu \mathrm{m})$ the impression of a seemingly homogeneous process. Both possibilities are realistic.

PEEM images the local work function with a resolution of $\approx 1 \mu \mathrm{m}$ but yields no or only indirect information about the chemical identity of the im- aged species. To obtain this chemical information we used SPEM. In SPEM measurements with the microstructured Pt/YSZ samples (Fig. 1b) a strong increase of the O1s signal on the Pt was observed upon electrochemical pumping with a positive voltage [8]. The shift of the O1s binding energy (BE) seen with increasing oxygen coverage from 530.4 to $530.0 \mathrm{eV}$ is comparable to that when oxygen from the gas phase is adsorbed. Thus, we were unable to detect a specific spillover species with properties different from that of chemisorbed oxygen. This finding is seemingly at variance with a previous report where a large shift of $\approx 1.6 \mathrm{eV}$ lower $\mathrm{BE}$ than that of chemisorbed oxygen was observed upon electrochemical pumping [14]. It is, however, well conceivable that these differences in $\mathrm{BE}$ just originate from different oxygen coverages which have been reached in the two sets of experiments.

\section{Conclusions and outlook}

It has been demonstrated that spatially resolving measurements help to reveal important mechanistic steps in the electrochemical promotion of catalytic reactions. In the case of a sodium-conducting solid electrolyte, already STM allowed the direct observation of sodium spillover species [15]. PEEM offers the possibility to observe local work function changes virtually in real time on a mesoscopic level. So far we were unable to detect any diffusion front of a possible spillover species, both on porous sintered $\mathrm{Pt}$ films and on microstructured thin $\mathrm{Pt}$ films. A moving reduction front, however, was observed with PEEM on the YSZ surface upon cathodic polarisation, indicating that the YSZ electrolyte might actively participate in the electrocatalytic effects observed with Pt/YSZ electrodes.

The measurements also demonstrated a strong dependence of the electrocatalytic behavior on the preparation of the samples. For the samples prepared at Patras, the WF more or less followed the variation of $V_{\mathrm{WR}}$ whereas for the samples with porous Pt layers prepared in our laboratory no WF changes at all were seen upon variation of $V_{\mathrm{WR}}$. The reason for this discrepancy is still unclear but one can suspect that factors which are difficult to control like the presence of chemical contaminants or oxides inside the $\mathrm{Pt}$ 
pores or the microstructure at the Pt/YSZ interface might be responsible.

Apparently more new questions have been raised by these results than can momentarily be answered. A systematic study of the effects of differently prepared samples by a combination of electrochemical and local spectroscopic methods under identical experimental conditions should finally lead to a real understanding of the origin of these differences.

\section{Acknowledgements}

The authors are indebted to C.G. Vayenas, Patras, for the supply of several Pt/YSZ samples used in their experiments. Financial support from the DFG (Deutsche Forschungsgemeinschaft) is gratefully acknowledged.

\section{References}

[1] C. Wagner, Adv. Catal. 21 (1970) 323.

[2] C.G. Vayenas, S. Bebelis, S. Ladas, Nature 343 (1990) 625.
[3] C.G. Vayenas, S. Bebelis, I.V. Yentekakis, H.G. Lintz, Catal. Today 11 (1992) 303.

[4] C.G. Vayenas, M.M. Jaksic, S.I. Bebelis, S.G. Nephytides, in: J.O.M. Bockris et al. (Eds.), Modern Aspects of Electrochemistry, Vol. 29, Plenum Press, New York, 1996, p. 57.

[5] C.G. Vayenas, Interface 1 (1996) 34.

[6] C.G. Vayenas, S.I. Bebelis, Solid State Ionics 94 (1997) 267.

[7] J. Poppe, A. Schaak, J. Janek, R. Imbihl, Ber. Bunsenges. Phys. Chem. 102 (1998) 1019-1718.

[8] B. Luerssen, S. Günther, H. Marbach, M. Kiskinova, J. Janek, R. Imbihl, Chem. Phys. Lett. 316 (2000) 337.

[9] J. Poppe, S. Völkening, A. Schaak, E. Schütz, J. Janek, R. Imbihl, PCCP 1 (1999) 5241.

[10] S. Völkening, Diploma thesis, Hannover, 1996.

[11] S. Ladas, S. Bebelis, C.G. Vayenas, Surf. Sci. 251-252 (1991) 1062.

[12] S.G. Neophytides, C.G. Vayenas, J. Phys. Chem. 99 (1995) 17063.

[13] B. Luerssen, in preparation.

[14] S. Ladas, S. Kennou, S. Bebelis, C.G. Vayenas, J. Phys. Chem. 97 (1993) 8845.

[15] M. Makri, C.G. Vayenas, S. Bebelis, K.H. Besocke, C. Cavalca, Surf. Sci. 369 (1996) 351. 